\title{
Bulk scaling of flow characteristics in the interior of sparse, emergent and rigid vegetation patch
}

\author{
Soumen Maji ${ }^{1,}$, and Prashanth Reddy Hanmaiahgari ${ }^{2}$ \\ ${ }^{1}$ Assistant Professor, Department of Civil Engineering, CIT Kokrajhar, Assam-783370, India \\ ${ }^{2}$ Assistant Professor, Department of Civil Engineering, IIT Kharagpur, West Bengal-721302, India
}

\begin{abstract}
Vegetation has an important role on erosion and sedimentation of rivers, river bank and marshy lands, etc. This effect depends on type of flow characteristics present in a vegetation patch. However, it's a great challenge to find out the flow characteristics in the interior of vegetation patch. The objective of this study is to determine the appropriate scaling of flow characteristics throughout the interior of an emergent and sparse vegetation patch for a given flowrate and depth, which can be used to predict the flow field in a similar vegetation conditions. In this study uniform acrylic cylinders were planted in a structured array to create a vegetation patch. Two different flow conditions by varying aspect ratio for a given Reynolds number were used in this laboratory study. Nortek ADV was used for measuring point velocities in the interior of the vegetation patch. Mean flow and turbulence quantities at all the measuring locations in the interior of the patch were scaled appropriately so that they collapse on a single curve. The local maximum velocity is found to be an appropriate scaling parameter for normalizing the streamwise velocity profiles, further the scaled velocity in the interior of the patch found to be following a power law. Lateral and vertical velocities in the interior of the patch are appropriately scaled by velocity vector across the section. Average bulk lateral velocity and scaled shear stress in a sparse and emergent vegetation patch can be described by linear equations in terms of scaled depth.
\end{abstract}

\section{Introduction}

Emergent vegetation in open channel flow has a great impact on the flow hydrodynamics and causes morphological changes. Therefore, studying emergent vegetation is important to determine its effect on velocity distribution and turbulence moments. Emergent vegetation has a relatively stiff stems, which are usually circular in cross section and without leaves below the water surface (O'Hare, 2015). Therefore, emergent rigid cylindrical stems have been extensively employed in following research works as a proxy for natural stems.

Meftah and Mossa 2013; Rubol et al. 2016 etc. studied flow characteristics in rigid vegetation patches. The rigid vegetation causes the flow to produce flow separation, wakes and eddies which in turn dissipate the flow energy (Ricardo et al., 2014). Vegetation may be considered as an obstruction to flow and a transversal sharp transition region is formed at the interface between the vegetation patch and flow (Meftah and Mossa 2013). In addition, they

* Corresponding author: s.maji@,cit.ac.in 
observed momentum transfer in the form of shear stress at the interface between obstructed and unobstructed flows. The vertical structure of flows within stems of emergent vegetation is divided in three layers: i) bottom layer, where the flow is highly $3 \mathrm{D}$ due to interaction with the bed; ii) the layer close to the free surface, which is affected by the oscillations of the free surface; and iii) the intermediate layer, sufficiently away from the bed and from the free surface, where the flow is controlled by the vertical stems and the flow properties are approximately constant in the vertical direction (Stoesser et al., 2010). Very small vertical velocities are observed in large spacing cases except just behind the cylinder where relatively large values of vertical velocity indicate considerable upward movement of fluid (Stoesser et al., 2010).

The objective of this study is to determine the bulk scaling of flow characteristics in an emergent and sparse vegetation patch for a given flowrate and depth, which can be used to predict the flow field in a similar vegetation conditions. The content of this research is given as following. First, a detailed description of experimental set-up is provided. Methodology of experimental data collected at various lateral locations in the interior of the vegetation patch is explained. Followed by post processing of measured data by using advanced filtering schemes is presented. Bulk scaling of streamwise velocity followed by $3 \mathrm{D}$ turbulent intensities and Reynolds shear stress has been discussed in detail. Finally, the summary of results obtained is collated.

\section{Experimental Set-up}

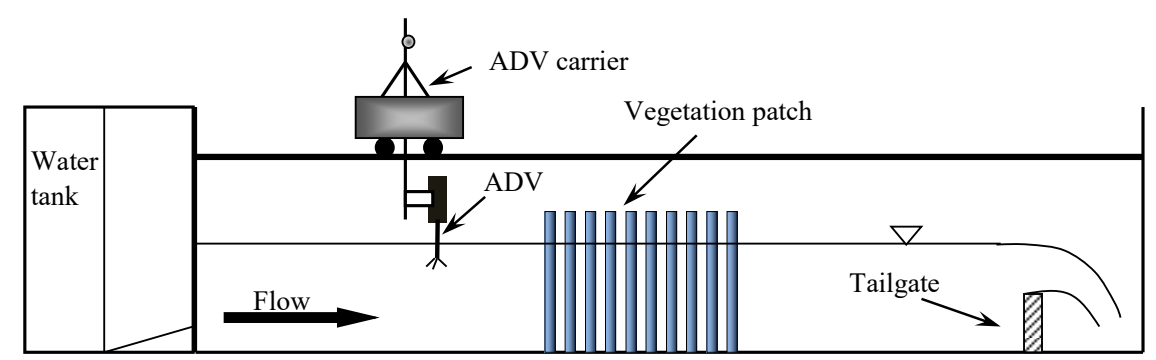

Fig. 1. Schematic diagram of the open channel turbulent flow with an emergent and sparse vegetation patch.

All experiments were performed in a wide rectangular channel $(1200 \mathrm{~cm}$ long, $91 \mathrm{~cm}$ wide and $61 \mathrm{~cm}$ deep) existing in the Hydraulic and Water Resources Engineering Laboratory of Indian Institute of Technology Kharagpur, India. The flume contained two glass sidewalls (800 cm long and $1.5 \mathrm{~cm}$ thickness) for the clear view of the flow. The test section of the flow was considered as $300 \mathrm{~cm}$ long which begins from $700 \mathrm{~cm}$ downstream of the inlet. At the inlet, a honeycomb baffle wall was provided to develop smooth, vortex free and uniform flow before reaching the test section. In the middle region of the test section as well as the cross-sectional area of the flume, an emergent, rigid and sparse vegetation patch was constructed by seventy uniform acrylic cylindrical rods $(0.64 \mathrm{~cm}$ diameter and $30 \mathrm{~cm}$ long) which were planted perpendicularly in a regular pattern on a perspex sheet fixed in the channel bed. The sparse nature of the vegetation was created using a $7 \times 10$ array of cylinders in which the spacing between the centers of two consecutive cylinders along streamwise and lateral directions were $9 \mathrm{~cm}$ and $4 \mathrm{~cm}$ respectively. The dimensions of the entire vegetation patch were $81 \mathrm{~cm}$ long and $24 \mathrm{~cm}$ wide and its solid volume fraction is 0.00894 . Sand particles of size $d_{50}=0.25 \mathrm{~cm}$ were coated on the flume bed to make the bed surface rough. A not to scale schematic diagram of the sparse vegetation patch placed in the middle of the flume is shown in Figure 1. At each measurement station as shown in Figure. 2, velocities were measured at many vertical locations. 


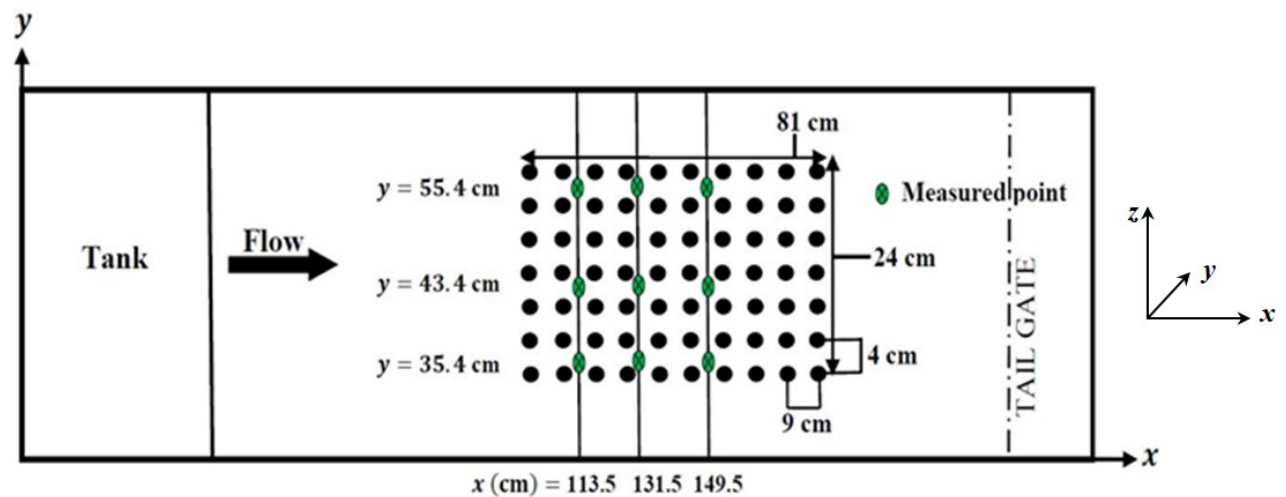

Fig. 2. Schematic diagram of the top view of the measurement stations along streamwise and lateral direction.

The flow of water was recirculated to and from the underground sump through the flume by two centrifugal pumps. A calibrated V-Notch was used to measure the flow rate entering the flume. For the measurement of instantaneous flow velocities, a three-dimensional Nortek Vectrino Plus acoustic Doppler velocimeter (ADV) was used which contained a fixed probe and four sideways looking beams. For smooth and convenient positioning of ADV, carriages were provided on rails with a track on the sidewalls of the flume. The water depth $h$ in the experiment was maintained as $15 \mathrm{~cm}$ and $20 \mathrm{~cm}$ by a tailgate which was fixed at the downstream end of the flume. The measurement of three-dimensional velocity components using ADV was started after half an hour of the commencement of the flow as the flow field seemed to be stabilized at this time. The cross-sectional lines through those streamwise locations were located in interior $(x=113.5,131.5,149.5 \mathrm{~cm})$ of the vegetation patch. At each streamwise location, vertical profiles of velocities were measured at three lateral stations which were $y=35.4,43.4$ and $55.4 \mathrm{~cm}$. Instantaneous velocities were measured at 15 points along the depth which were $0.3,0.5,0.7,0.9,1.5,2,2.5,3,4,5,6,7,8,9$ and $10 \mathrm{~cm}(12 \mathrm{~cm}$ and $15 \mathrm{~cm}$ when water depth was $20 \mathrm{~cm}$ ) from the channel bed at each station. Figure 2 shows a not to scale top view of the streamwise and lateral locations where experimental data was measured. Table 1 shows the different flow conditions in the present laboratory experiments, in which, $h=$ flow depth; $\bar{u}=$ time-averaged streamwise velocity; $u_{\max }=$ maximum streamwise velocity; $u_{*}=$ shear velocity; $R_{e}=$ Reynolds number; $F_{r}=$ Froude number and $A_{r}=$ Aspect ratio.

Table 1. Different flow conditions used for laboratory experiment

\begin{tabular}{|l|l|l|l|l|l|l|l|}
\hline Exp. & \multicolumn{1}{|c|}{$\begin{array}{c}\boldsymbol{h} \\
(\mathrm{m})\end{array}$} & $\begin{array}{c}\overline{\boldsymbol{u}} \\
(\mathrm{m} / \mathrm{s})\end{array}$ & $\begin{array}{c}\boldsymbol{u}_{\max } \\
(\mathrm{m} / \mathrm{s})\end{array}$ & $\begin{array}{c}\boldsymbol{u}_{*} \\
(\mathrm{~m} / \mathrm{s})\end{array}$ & \multicolumn{1}{|c|}{$\boldsymbol{R}_{\boldsymbol{e}}$} & \multicolumn{1}{|c|}{$\boldsymbol{F}_{\boldsymbol{r}}$} & \multicolumn{1}{|c|}{$\boldsymbol{A}_{\boldsymbol{r}}$} \\
\hline $\operatorname{Re} 1 \mathrm{Ar} 1$ & 0.155 & 0.29 & 0.30 & 0.238 & 43500 & 0.235 & 5.87 \\
\hline $\operatorname{Re} 1 \mathrm{Ar} 2$ & 0.20 & 0.22 & 0.25 & 0.197 & 43500 & 0.157 & 4.55 \\
\hline
\end{tabular}

\section{Data Processing}

For the post processing of data obtained from the ADV, a three-dimensional coordinate system has been adopted where $x, y$ and $z$ denoted as streamwise, transverse and vertical directions respectively. The coordinate system can be well understood from Fig. 2. Spike filtering, correlation and signal-to-noise ratio (SNR) thresholding techniques were used as 
suggested by Chanson et al. (2008) to minimize the effect of the Doppler noise in the data. To further improve the accuracy of the turbulence measurements, low correlations and low SNR data were removed. The basic ideas of Goring and Nikora (2002) for removing the random spikes were implemented. The spikes in velocity measurements were removed from the raw time series. The number of spikes consisted less than $5 \%$ of the original velocity record and had no effect on the spectrum and overall frequency content. Velocities along $x$, $y$ and $z$ directions are denoted as $u, v$ and $w$. The fluctuation parts of $u, v$ and $w$ are represented by $u^{\prime}, v^{\prime}$ and $w^{\prime}$ respectively.

\section{Results and Discussion}

Two experiments have been conducted in this study and corresponding experimental flow conditions are given in the Table. 1. Experimental data were collected at upstream, interior and wake region along the streamwise and lateral directions of the flow, however analysis of interior flow only is presented in this paper. All experimental data were plotted together to investigate the self-similarity of velocity and other turbulence parameters of flow field and which is called bulk scaling in this study.

\subsection{Scaling of streamwise velocity}

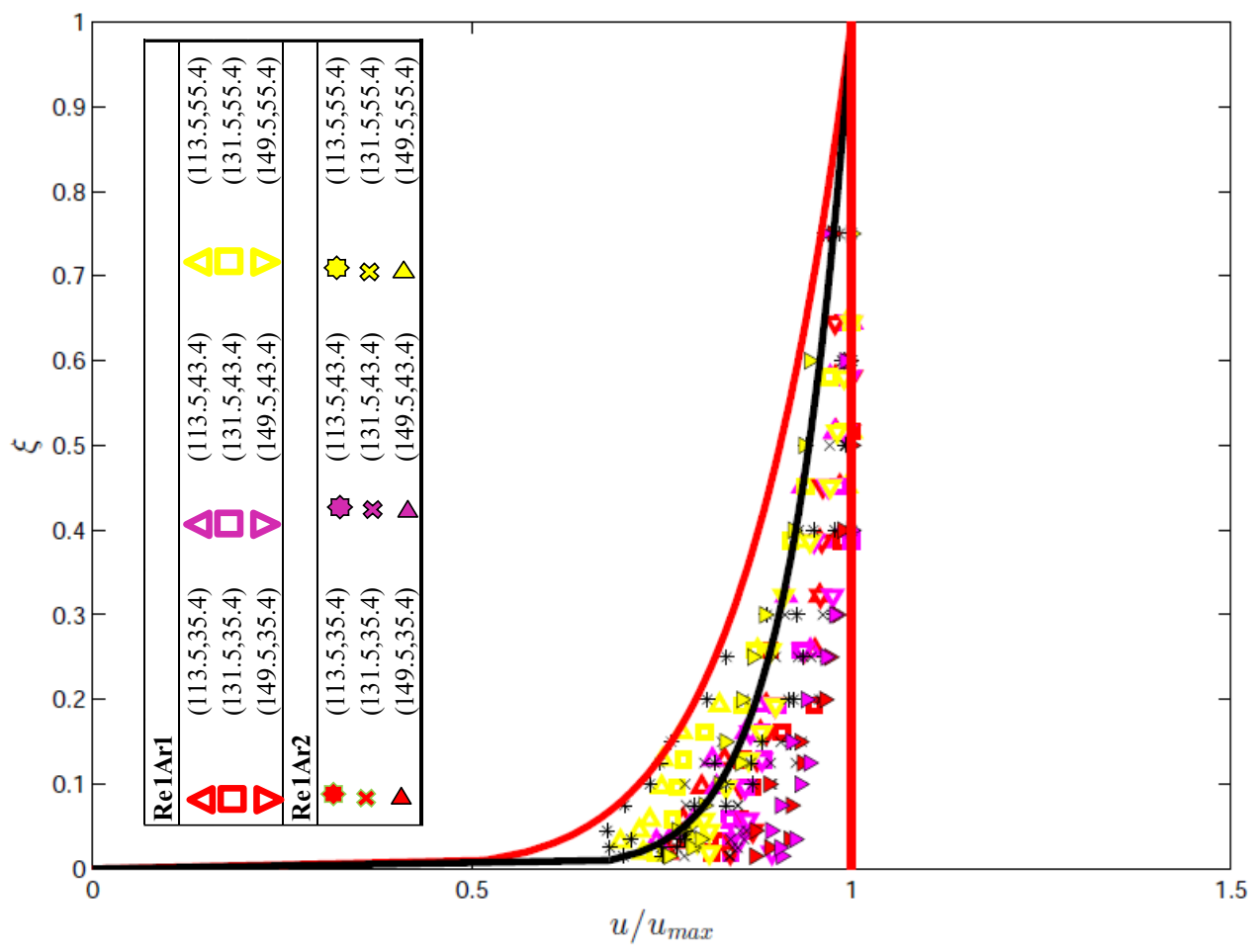

Fig. 3. Streamwise velocity distribution profile (non-dimensionalized with average velocity $u_{\max }$ ).

Fig. 3 demonstrates that streamwise velocity profile in sparse and emergent vegetation patch is following a power law (Fig. 3). Fig. 3 shows the maximum velocity $u_{\max }$ is appropriate for the velocity scaling and flow depth $(h)$ as a characteristic length scale. It is inferred from Fig. 3 that changes in aspect ratio don't affect the self-similarity. It is known that the power 
law, in the form of $u / u_{\max }=(\xi)^{1 / m}$, with $m$ varying from 6 to 7 , may largely approximate the velocity profiles observed in open channel flows without vegetation (Cheng et al. 2012). However, the spread of the data points plotted in Fig. 3 does not support the power law with $m$ values in the narrow range. The velocity profiles are roughly confined by the two power laws, with $m=7$ and a vertical line. Interior of the patch, exponent of velocity power law increases with increase in downstream distance. At the leading edge of the vegetation patch, velocity profile best described by the power law with $m=7$ (refer to Fig. 3). However, the velocity profile at the trailing edge is described by the vertical line which is due to the attainment of complete mixing of the flow necessitated by the availability of enough space for the formation of vortices, stretching and interacting with other vortices causing uniform velocity.

\subsection{Scaling of turbulence intensities}

\subsubsection{Streamwise turbulence intensity}

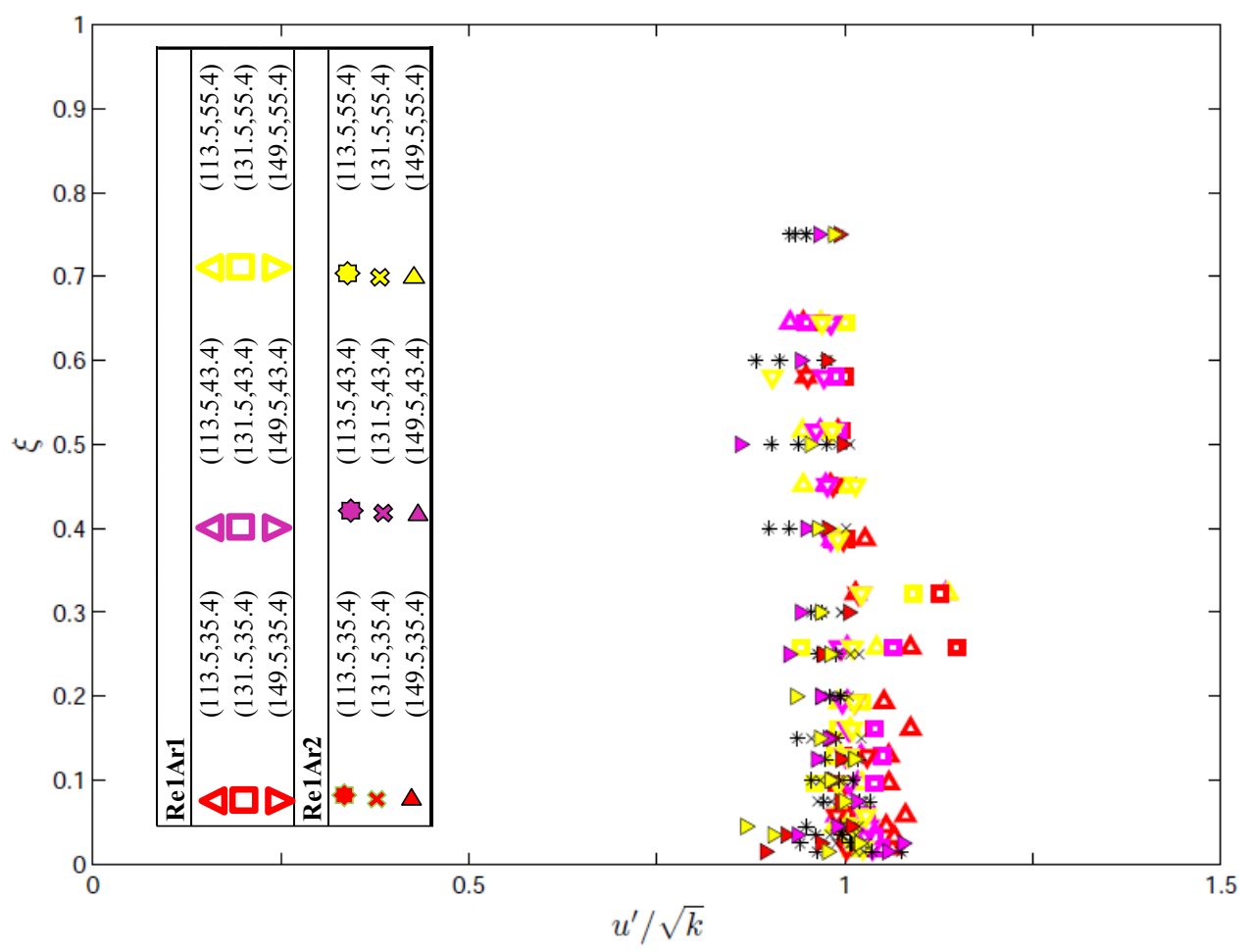

Fig. 4. Vertical distribution of streamwise turbulence intensity.

Vertical distribution of streamwise turbulence intensity has been plotted in the Fig. 4 for the two experimental data. Streamwise turbulence intensity has been normalized by square root of turbulent kinetic energy $(\sqrt{k})$ in these plots. Fig. 4 demonstrates that $u^{\prime} / \sqrt{k}$ profile is uniform and rectangular in shape. No effect of aspect ratio on streamwise turbulence intensity has been noticed by changing the aspect ratio in the experiments. The magnitude of $\left(u^{\prime} / \sqrt{k}\right)$ found to be varying from 0.9 to 1.15 . 
4.2.2. Lateral turbulence intensity

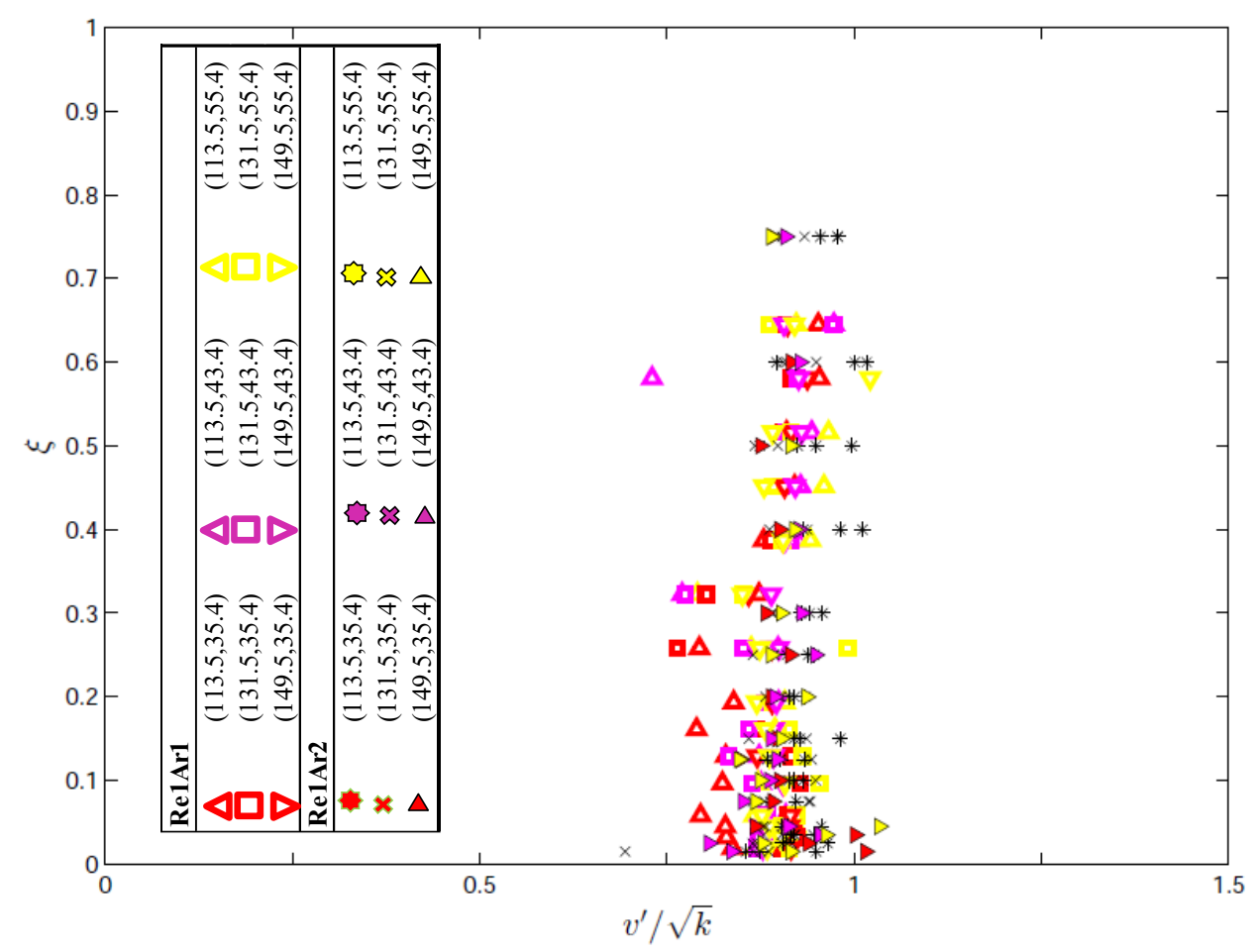

Fig. 5. Lateral turbulence intensity distribution profile.

Non-dimensionalized lateral turbulence intensity distribution profile has been shown in Fig. 5. It can be seen similar to streamwise turbulence intensity (fig. 4), vertical fluctuation distribution profile is also uniform and rectangular in shape. The magnitude of $\left(v^{\prime} / \sqrt{k}\right)$ is varying from 0.75 to 1.05 . Although distribution of lateral velocity is similar irrespective of aspect ratio, the magnitude of $\left(v^{\prime} / \sqrt{k}\right)$ is found to be lesser in the case of lower aspect ratio flow than high aspect ratio flow.

\subsubsection{Vertical turbulence intensity}

Vertical turbulence intensity profile has been shown in Fig. 6, which shows that vertical turbulence intensity profile follows a power law in the interior of the vegetation. Lower magnitude of $\left(\mathrm{w}^{\prime} / \sqrt{\mathrm{k}}\right)$ is recorded near bed throughout the cross section and the maximum magnitude is found in the outer layer. The magnitude of $\left(\mathrm{w}^{\prime} / \sqrt{\mathrm{k}}\right)$ is varying between 0.35 and 0.51 .

\subsection{Scaling of Reynolds shear stress}

Non-dimensionalized Reynolds shear stress profiles in the interior of the vegetation patch have been shown in Fig. 7. The maximum value of $\left(-\overline{u^{\prime} w^{\prime}} / u_{*}^{2}\right)$ has been observed in the inner layer. The normalized values are deviating (red lines) from the expected line (black line). Unlike the fully developed flow where the maximum value of $-\overline{u^{\prime} w^{\prime}}$ profiles occur near the channel bed, in the latter half of the vegetation patch, the peak in the RSS profiles is 


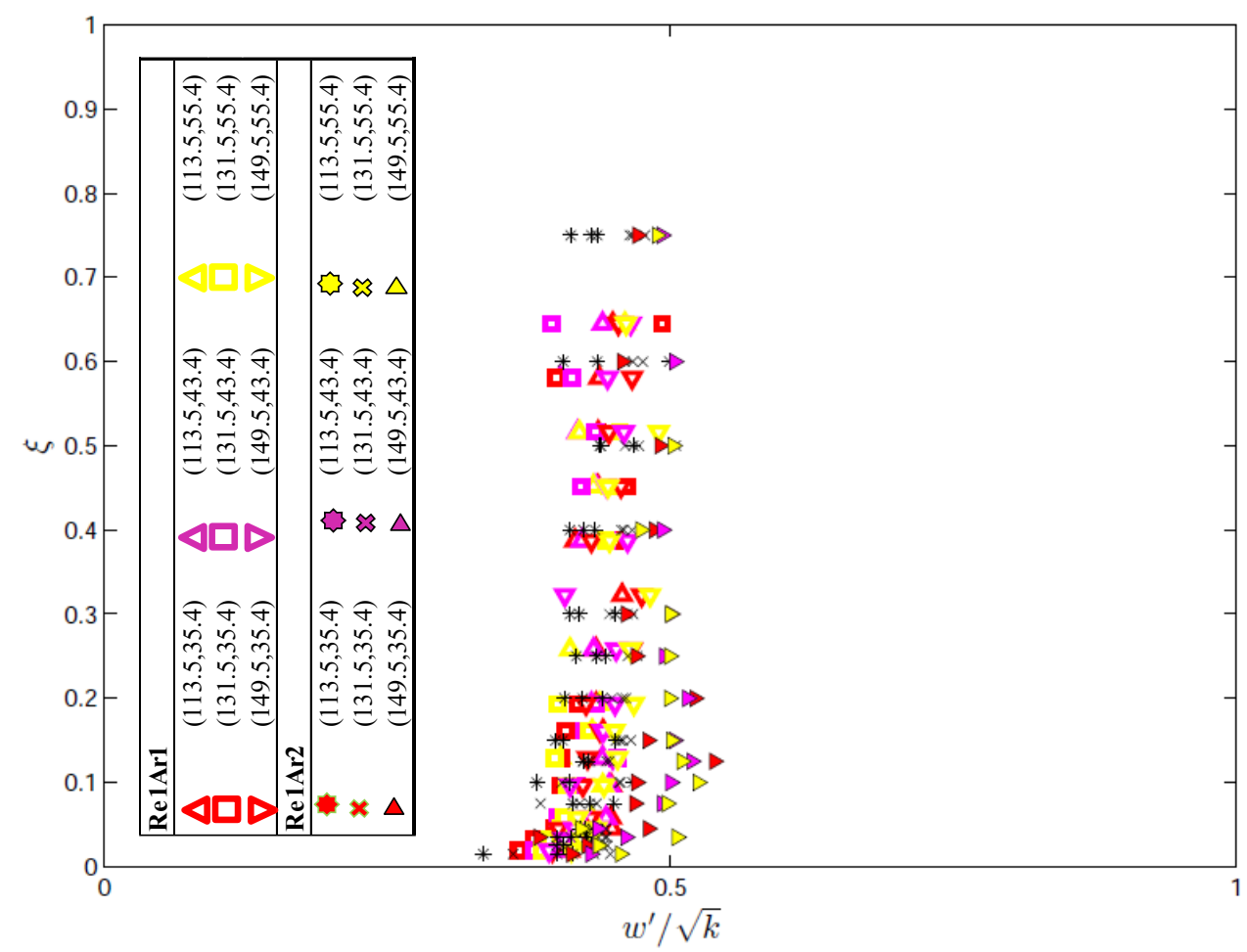

Fig. 6. Vertical turbulence intensity distribution profile.

occurring in the intermediate layer due to the wall wake effect. The $-\overline{u^{\prime} w^{\prime}}$ distributions interior vegetation patch falling below the expected line. However, $-\overline{u^{\prime} w^{\prime}}$ distribution is higher in non-obstructed area (without vegetation) due to the flow diversion through that region therefore falling above the expected line.

The total distribution is encapsulated in between the lower and upper boundaries as shown in Fig. 7. An average of these boundaries gives bulk average of Reynolds stress distribution of flow through emergent, sparse and rigid vegetation patch. Hence, following linear equation (Eq. 1), defines the relation between Reynolds shear stress and vertical depth of flow for this case and similar flow fields. The Eq. 1 says that Reynolds stress distribution in sparse and emergent vegetated flow is $85 \%$ of fully developed flow without vegetation.

$$
\frac{\tau}{\rho u_{*}^{2}}=0.85(1-\xi)
$$

\section{Conclusions}

Laboratory experiments were conducted and measurements were taken along streamwise and lateral directions in the interior of the emergent and sparse vegetation patch with two aspect ratios. Mean flow and turbulence quantities at all the measuring locations in the interior of the patch were scaled appropriately so that they collapse on a single curve. The maximum velocity is suggested as the appropriate scaling parameter for normalizing the streamwise velocity profiles within the vegetation patch. Similarly, flow depth is the length scale. The velocity profiles are roughly confined by the two power laws, with power 7 and a vertical line. At the leading edge of the vegetation patch, velocity profile best described by the power law with power 7, and the exponent increases as streamwise distance increases interior of the patch. However, the velocity profile at the trailing edge is described by the vertical line. 


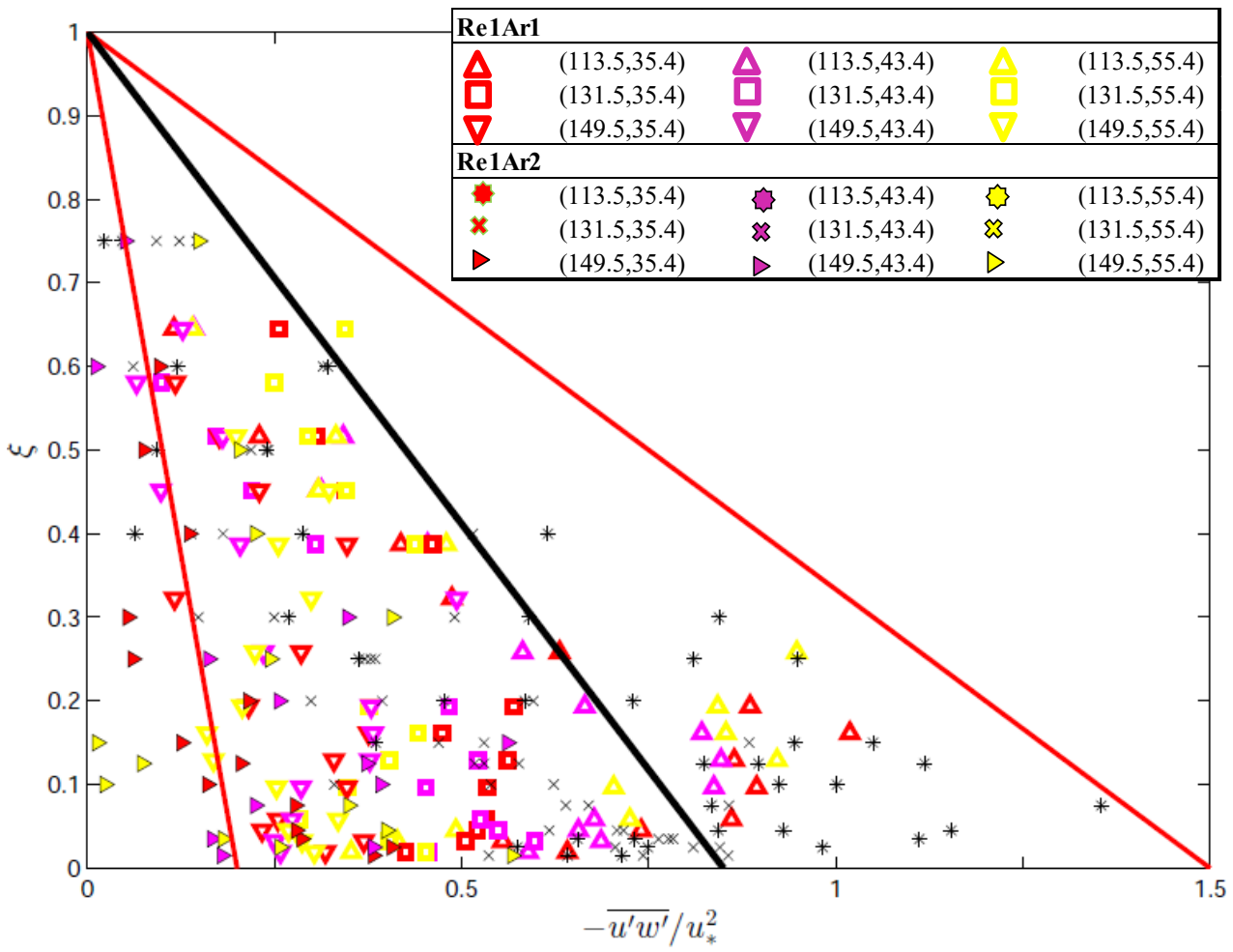

Fig. 7. Reynolds shear stress distribution profile.

Turbulence intensities $\left(u^{\prime}, v^{\prime}\right.$ and $\left.w^{\prime}\right)$ scaled by the square root of kinetic energy are linear. The bulk average values are in the range of 0.9-1.15, 0.75-1.05 and 0.35-0.6 respectively for $u^{\prime}, v^{\prime}$ and $w^{\prime}$. Bulk average of scaled Reynolds shear stress distribution in sparse and emergent vegetated flow can be described by $85 \%$ of the RSS linear distribution in the fully developed flow without vegetation.

\section{References}

1. Chanson, H., M. Trevethan and S. I. Aoki, Acoustic doppler velocimetry (adv) in small estuary: field experience and signal post processing. Flow Measurement and Instrumentation 19(5), 307-313, (2008).

2. Goring, D. G. and V. I. Nikora, Despiking acoustic doppler velocimeter data. Journal of Hydraulic Engineering 128(1), 117-126, (2002).

3. Meftah, M. B., and Mossa, M. Prediction of channel flow characteristics through square arrays of emergent cylinders. Physics of Fluids (1994-present), 25(4), 045102, (2013).

4. O'Hare, M. T. 'Aquatic vegetation - a primer for hydrodynamic specialists', Journal of Hydraulic Research. Taylor and Francis, 53(6), pp. 687-698. (2015).

5. Ricardo, A. M., Koll, K., Franca, M. J., Schleiss, A. J. and Ferreira, R. M. L. 'The terms of turbulent kinetic energy budget within random arrays of emergent cylinders', Water Resources Research, 50(5), pp. 4131-4148, (2014).

6. Rubol, S., Battiato, I., and de Barros, F. P. Vertical dispersion in vegetated shear flows. Water Resources Research, 52(10), 8066-8080, (2016).

7. Stoesser, T., Kim, S. J. and Diplas, P. 'Turbulent flow through idealized emergent vegetation', Journal of Hydraulic Engineering, 136(12), pp. 1003-1017,(2010). 